\title{
The Operation and Feedback Mechanism of Building Construction Safety Supervision Management Mode
}

\author{
BuXiangyi \\ Wuhan University of Technology,Wuhan 430070,China
}

\begin{abstract}
Key words: building construction; safety supervision; feedback mechanism; coordination mechanism; system simulation
\end{abstract}

\begin{abstract}
From the definition of building construction safety supervision management system, the theoretical framework of construction safety supervision and management cooperative feedback mechanism has been proposed, and the supervision management of cooperative feedback mechanism level and basic functions has been analyzed. The double closed loop feedback control system based on the safety of development unit and construction unit is proposed, which makes the reaction speed of the feedback regulation mechanism more sensitive. When faced with safety problems, the recovery rate is fast. The operation mechanism of building construction safety supervision management system are studied in this paper, analysis of the quartet coordination mechanism of government construction administrative department, building industry associations, development units and construction unit. Finally, the system simulation of the construction safety supervision management feedback mechanism is carried out, it can be seen from the simulation results that, construction safety management level is higher after using a feedback mechanism, which provides an important theoretical reference for operation and feedback mechanism study of construction safety supervision management mode.
\end{abstract}

\section{Introduction}

In recent years, although the construction industry safety management level has been continuously improved in China, the major safety accident of construction projects still occur, economic losses and casualties are huge, having extremely bad social impact. There are many factors that cause construction safety management problems, but the inadequate of supervision system is a major cause of construction safety accidents. Therefore, it is an important way for effective prevention and control of construction safety accident reflex how to mobilize government construction administrative department, building industry associations, supervision units and construction units and the whole society to jointly participate in the enthusiasm of the safety supervision management, and build perfect construction management and feedback mechanism, which is significant important for the study of construction safety production in China.

\section{Construction safety supervision management cooperative feedback mechanism}

In the market economy management system, the construction project participants mainly include development units, construction units and supervision units, they will maximize the interests of their own in the process of engineering construction. The pursuit of construction unit is to gain the maximum profit in the shortest time, the pursuit of construction units and supervision units is fast speed, good quality and low cost. They tend to make the behavior that is not conducive to the safety management in the pursuit of their own interests, so it is very difficult to realize the balance of interest simply by mutual supervision of enterprises. In order to avoid the occurrence of safety accidents in construction at the greatest degree, and ensure the smooth progress of the construction project and the long-term development of the construction industry, it is needed that government construction administrative departments and construction industry associations balance the interests of all parties in the macroscopic angle, and constrain the construction units and the construction units according to the safety related legal rules, and finally form tetragonal coordination feedback mechanism. 


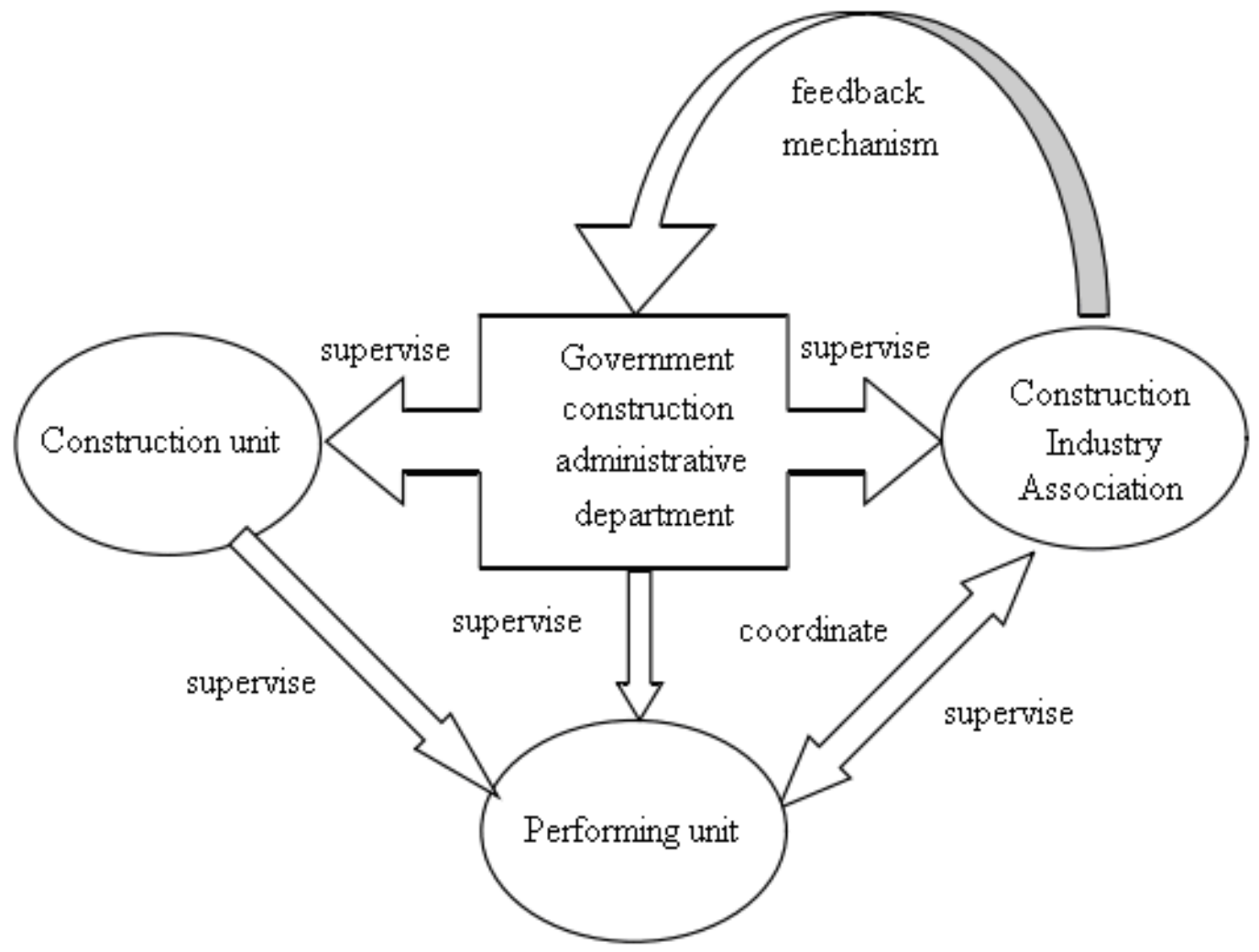

Figure 1 The block diagram of construction safety supervision management collaborative feedback mechanism

Figure 1 shows the block diagram of construction safety supervision management collaborative feedback mechanism, which includes three functions of supervision, coordination and feedback.

(1) Supervision function

The government construction administrative departments are mainly responsible for the implementation of the laws and regulations on the safety of the development units and the construction units, and management on safety behavior, and providing the service of the necessary construction guide and organize safety education activities for them. It supervises the management of the construction industry association, and directly supervise and manage the construction unit. The development unit is an important participant in building engineering activities, it plays a very important role for the safety supervision of the construction unit.

(2) Coordination function

The construction industry associations have function of coordinating that cooperate with government construction administrative departments, construction units, supervision units and development units, which can actively promote an equal consultations on construction units and development units and achieve the interests balance between them. The construction industry association, with fairness and justice, is the fourth organization that is independent of the government, development unit and construction unit, which plays an important role to coordinate the third party and deal with the construction unit and construction unit dispute, and promote the communication and contact.

(3) Feedback function

According to problems in the process of supervision and management of the construction unit, the construction industry associations can feedback to the government department, and urge government departments to react in the shortest time, and speed up the information flow, and improve the supervision and management efficiency of the government.

\section{Safety supervision management mechanism based on double closed loop feedback}


In the construction process, when suddenly encountered security problems, it will cause the interests dispute between the development units, if directly use the construction unit to feedback the security problems; if directly use the development units to feedback the security problems, it will cause the interests problem of the construction unit. In view of the above problems, double closed loop feedback control system based on the security problems of the development units and construction units has been proposed in this paper, and the reaction velocity of the feedback regulation mechanism is more sensitive. When encountered security problems, the reply speed is faster.

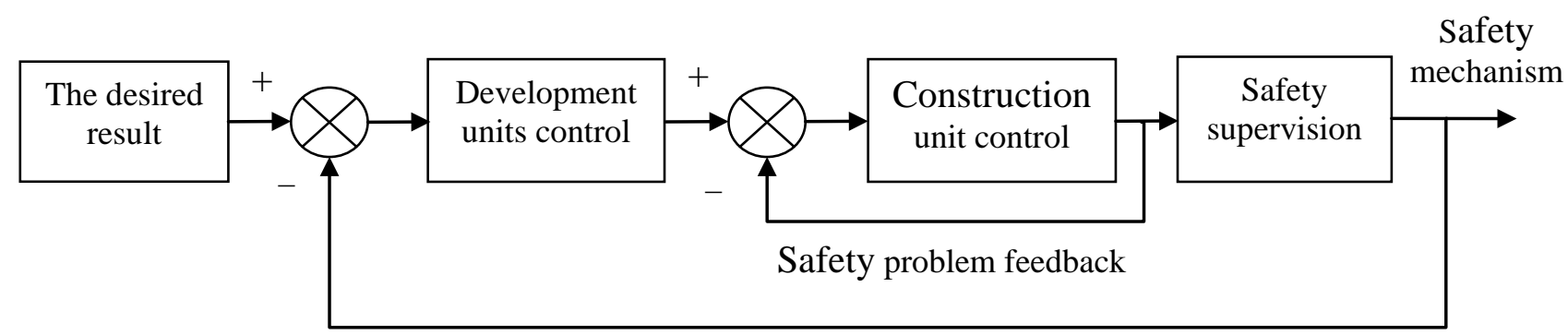

Fig.2 The double closed loop control schematic of building construction safety supervision

The double closed loop PID control of construction safety supervision can be shown in Figure 2, safety supervision and control can be carried out taking construction unit as the outer ring, and development units as the inner loop, positional digital PID control can be used in the PID control algorithm of safety supervision.

$$
u(t)=k_{p} e(t)+k_{i} \int_{0}^{t} e(t) d t+k_{d} \frac{d e(t)}{d t}
$$

In the formula (1), $u(t)$ is the PID output value, $e(t)$ is the error between the actual and desired output values, $\int_{0}^{t} e(t) d t$ is integral quantity, $\frac{d e(t)}{d t}$ is differential quantity, $k_{p}, k_{i}$ and $k_{d}$ are proportional, integral and differential coefficient, and PID calculation formula can be obtained by discretion of integral quantity and differential quantity.

$$
u(t)=k_{p} e(t)+k_{i} \sum_{j=0}^{t} e(j) T+k_{d} \frac{e(t)-e(t-1)}{T}
$$

In the formula (2), $T$ is update time, PID control algorithm of Construction unit

$$
\begin{aligned}
& A P I D(\text { out })=k_{p} e(t)+k_{i} \sum_{j=0}^{t} e(j) T+k_{d} \frac{e(t)-e(t-1)}{T} \\
& A R a t e P I D(\text { out })=k_{p} e_{1}(t)+k_{i} \sum_{j=0}^{t} e_{1}(j) T+k_{d} \frac{e_{1}(t)-e_{1}(t-1)}{T}
\end{aligned}
$$

Formula (3) is the PID calculation formula of construction units loop, Formula (4) is the PID calculation formula of development units loop. APIDOut $(t)$ is the PID output of construction unit loop, ARatePIDOut $(t)$ is the PID output of construction units loop, $e(t)=$ expected value - actual value, $e_{1}(t)=\operatorname{APIDOut}(t)$ - the actual angular speed.

System dynamics (SD) is was founded by Jay W. Forrester in Massachusetts Science Institute of Technology (MIT) in 1956, It has been widely used in biology, ecology, energy, environment, economy, transportation, medical and other fields until now. In System Dynamics, the system is combination of features, elements in a collection exist mutual influence and organic link together through interactions, using combination of qualitative and quantitative processing to deal with complex system problems, which is the subject that study feedback.

The influence Analysis of the construction safety management feedback mechanism on safety management level 
The principle of system dynamics is used in this study according to the influence of the development units, the construction units and the government administrative department on the construction safety supervision management. double closed loop feedback control mechanism in the second section have used for the system simulation, the simulation framework is shown in Figure 3.

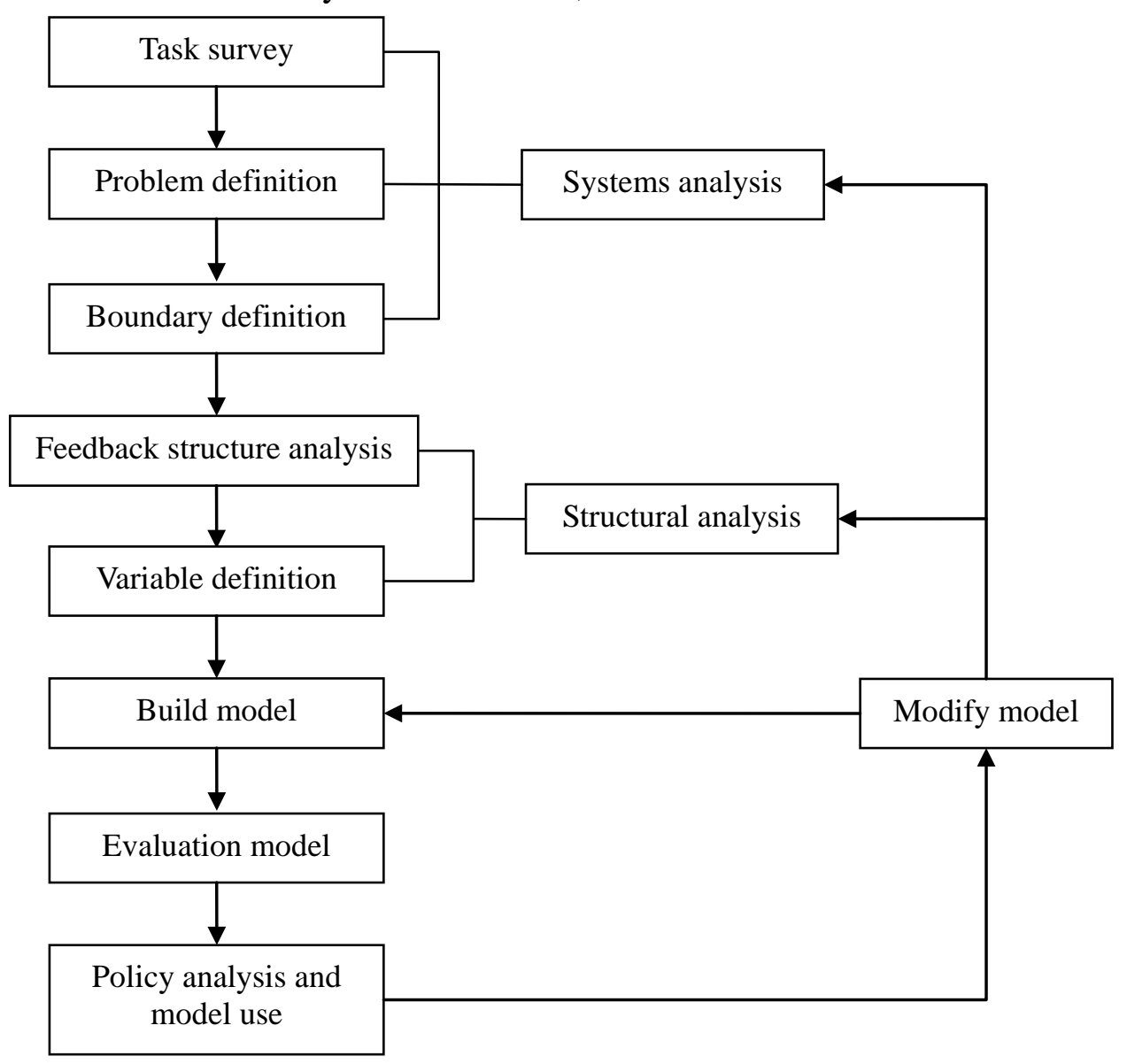

Fig. 3 System simulation framework

Figure 3 shows the simulation framework of construction safety supervision management system, in order to make the results more true, the feedback loop is divided into 8 loops, which is shown as follows.

Loop 1: development unit management level $(+)$ - construction site production safety management level (-) - development unit management level;

Loop 2: government construction administrative department supervision level $(+)$ - construction units safety level (-) - government construction administrative departments supervision level

Loop 3: construction unit safety construction level $(+)$ - construction site production safety management level (-) - construction unit safety construction level

Loop 4: supervision unit management level $(+)$ to the construction site production safety management level (-), supervision unit management level

Loop 5: construction unit safety design level (+) - construction site safety production level (-) construction unit safety design level

Loop 6: supervision unit management level $(+)$ - construction unit safety construction level $(+)$ construction site production safety management level

Loop 7: construction unit safety design level $(+)$ - supervision unit management level $(+)$ construction site safety production level (-) - construction unit safety design level

Loop 8: construction unit safety design level $(+)$ - construction unit safety construction level $(+)$ construction site safety production level (-) - construction unit safety design level 


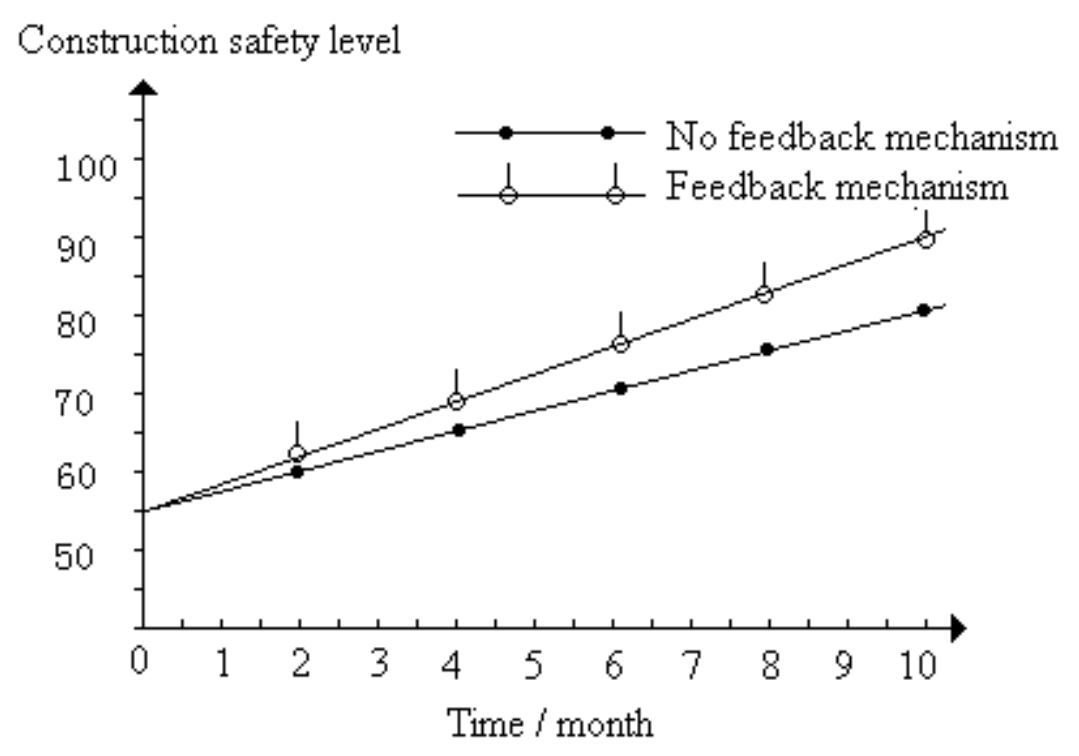

Fig. 4 The security level before and after using the security feedback mechanism

Figure 4 shows safety level that before and after using the safe feedback mechanism which was calculated by the simulation system, it can be seen from the figure that before and after the using safety feedback mechanism, with the increase of time, level of production safety in construction engineering are improved, but use safety feedback mechanism in the construction safety management level to obvious than does the use of feedback mechanism of high, thus validating the safety feedback mechanism in the construction safety supervision management role.

\section{Conclusion}

The quartet coordination mechanism of government construction administrative department, building industry associations, development units and construction unit has been in-depth analyzed in this study, and the double closed loop feedback control system based on the security problems of the construction units and development units has been put forward, the reaction speed of safety supervision and management mechanism is more sensitive by using the system. Simulation system of safety supervision and management mechanism has been carried out, it can be found from the simulation results that safety management level that using the construction safety management feedback mechanism is higher than that not using feedback mechanism, so the important role of the security feedback mechanism in the management of construction safety supervision is verified.

\section{Reference}

[1] Li Shirong, Bi Mo. Research and practice of construction safety management in the United Kingdom [J]. Journal of Engineering Management.2011,25 (4): 368-372.

[2] Zhao Yuejie. Talk about the government's supervision and management of construction safety [J]. Shanxi Architecture.2012.38 (27): 279-280

[3] Xu Zhengquan, Song Xuefeng, Xu Jinbiao. 4 leap of accident origin theory and its significance [J]. Mining Safety \& Environmental Protection, 2008, 35 (1):79-82.

[4] Zhang Shilian, Pan Chengshi. Research on safety design and construction safety in the design stage of construction project [J]. Construction Economy.2006.279 (1):77-80.

[5] Chen Baochun. Based on the comparison from the perspective of government regulation of construction safety analysis and countermeasures [J]. Journal of Safety Science and Technology, 2011.7 (3): 89-92. 
[6] Liu Zheng. Quantitative analysis on the influence of construction units on the safety of engineering projects [J]. Journal of Hebei University Of Science and Technology, 2011 (03):297-302.

[7] Zhou Quan, Fang Dongping. An Empirical Study on the influence mechanism of construction industry safety atmosphere on safety behavior [J]. China Civil Engineering Journal:129-132 (11).2009,42

[8] Zeng Yuzhong. The problems and analysis of the construction safety management [J]. Guangdong Science \& Technology.2008 (4): 195-196.

[9] Shen Huihua. Analysis of construction safety production management law perfect [J]. Science and Technology Innovation Herald. 2007 (26):72.

[10] Yang Caixia. Construction enterprise safety culture construction [J]. Journal of Beijing University of Civil Engineering and Architecture: 2009 (1),75-76.

[11] Cai Yanling. The construction enterprise safety culture construction [J]. China Science and Technology Information. 2008 (20):179-180.

[12] Xie Faquan. Supervision and management of construction safety [J]. Work Safety \& Supervision. 2009 (2):24-25.

[13] Pan Chengshi, Zhang Shilian. The significance of safety management to construction project management[J]. Building Economy, 2003, 24 (12): 43-44

[14] Fang Dongping, Wu Shenghou, Bi Shutao etc.. Scaffold accidents caused by cause analysis and Countermeasures of safety management[J]. China Civil Engineering Journal(Construction Engineering and Management Sciences, 2002.8. 25-30

[15] Zhao Tingsheng, Lu Xuewei, Fang Dongping. Investigation and analysis of the incentive factors of construction accidents[J]. Construction Technology, 2003.12 (33): 56-60

[16] Zhao Tingsheng, Fang Dongping, Wang Zehui. Construction quality and safety control of concrete construction[J]. Construction Technology, 2005.04 (35): 24-26

[17] Zhang Li, He Xueqiu. Application of safety rheology theory in mine accidents[J]. China safety Science Journal,.2001,11 (1): 6-11

[18] Zhang Xin, Mao Baohua. Analysis of traffic accident process based on the theory of safety rheology[J]. China safety Science Journal, 2004, 14 (1): 18-22 